\title{
Multiple Channel Integration Quality Assessment Method Using NARX
}

\author{
Xiaolei Wang $\mathbb{D}^{1}$ and Yingzhao He $\mathbb{C}^{2}$ \\ ${ }^{1}$ School of Economic and Management, Beijing Jiaotong University, Beijing 100044, China \\ ${ }^{2}$ School of Business, Northwest Normal University, Lanzhou 730070, China \\ Correspondence should be addressed to Xiaolei Wang; 12113148@bjtu.edu.cn
}

Received 20 October 2020; Revised 1 November 2020; Accepted 7 November 2020; Published 21 November 2020

Academic Editor: Abd E. I.-Baset Hassanien

Copyright (C) 2020 Xiaolei Wang and Yingzhao He. This is an open access article distributed under the Creative Commons Attribution License, which permits unrestricted use, distribution, and reproduction in any medium, provided the original work is properly cited.

\begin{abstract}
To improve the accuracy of the multiple channel integration quality (MCIQ) evaluation, this paper proposes a comprehensive evaluation method using the nonlinear autoregressive exogenous model (NARX) and constructs an index system. First, the entropy method is used to determine the objective weight of each indicator. The indicators used in this paper are process consistency, information consistency, emotional value, procedural value, service structure transparency, online result value, business relevance, and online purchase intention. Second, an improved gray relational analysis (GRA) algorithm is used to obtain the comprehensive gray relational degree between the above eight indicators' standard samples and the tested samples. Then, this study uses the dataset preprocessed with the GRA algorithm for training the NARX model. Then, this study uses the trained model to evaluate the quality of multiple channel integration comprehensively. Next, this study uses standardized methods to quantify the evaluation results to provide new ideas and theoretical guidance for teaching traditional retailers to use the advantages of multiple channels to expand their online business. This paper uses 50,000 consecutive samples of a product for 3 months as a dataset in the experimental part. Through the GRA method and the NARX model, the comprehensive gray relational degree between the test sample and the ideal sample is obtained, and the results are quantified. Experiments show that, compared with the GRA method, this paper's method has a higher degree of fit between the output value and the target value.
\end{abstract}

\section{Introduction}

With the rise and development of Internet companies, more and more "online shops" have emerged. Compared with traditional offline sales, online sales have gained more momentum in recent years, and there are even online "fitting rooms." To comprehensively analyze the influencing factors of consumers' purchase intentions and put forward valuable suggestions for traditional offline retail stores, we used the NARX neural network to conduct multichannel integration of customer purchasers' intentions and obtained corresponding results.

At present, many scholars have done some research on the quality of multiple channel integration. Berman et al. [1] believed that an excellent multiple channel integration strategy has the following characteristics: highly integrated promotion, cross-channel product consistency, shared customer integrated information system, cross-channel pricing and inventory information, online purchase and offline delivery service process, and provides multiple channel opportunity searches for suitable people. Saeed et al. [2] believed that the information system integration of retailers' virtual and physical channels can be divided into content integration, information integration, and logistics integration according to the value-added services provided. Chan et al. [3] showed that the systemic relationship between online and offline channels includes four dimensions: reinforcement, collaboration, interaction, and how much they complement each other. Lee et al. [4] divided the practice and effectiveness of retailers' cross-channel integration into five dimensions: information consistency, channel selection freedom, e-mail marketing effectiveness, 
channel interaction, and store customer service evaluation. Oh et al. [5] divided the retail process integration into six dimensions: commodity and price information integration, promotion information integration, transaction information integration, information acquisition integration, order fulfillment integration, and customer service integration. There are two manifestations of retailers' multiple channel integration. From the process's perspective, multiple channel integration is the comprehensive cross-channel allocation and function optimization of various retail portfolio factors. The service structure level and interaction consistency of the channel have been improved. Sousa et al. [6] proposed the concept of MCIQ and believed that multiple channel service quality includes three components: physical, virtual, and integrated service quality (i.e., MCIQ). Among them, MCIQ reflects the overall result of the multiple channel integration framework. Through in-depth interviews with consumers, it is found that consumers are more likely to make explicit judgments about multiple channel integration [7]. Therefore, this study measures multiple channel integration from the perspective of results.

Recently, there are many classifications of quality evaluation methods for multiple channel integration, but they all have limitations, such as low accuracy and cumbersome calculation processes. To improve MCIQ evaluation's accuracy and overcome the main shortcomings of existing methods, this paper proposes a comprehensive evaluation method of MCIQ using the NARX model. This paper is mainly divided into four parts. Firstly, the objective weight value of each indicator is determined according to the entropy method. Secondly, the improved GRA method is used to obtain the comprehensive gray correlation degree. Third, the NARX model is trained using the dataset processed by the GRA method. Then, the trained NARX model is used to predict the comprehensive gray correlation degree. Finally, the results calculated by the GRA method and NARX model are standardized and compared with the target value, and the quantitative results of MCIQ are given.

\section{Literature Review}

2.1. Multiple Channel Integration Quality and Its Assessment Method. Sousa et al. [6] proposed the concept of MCIQ and believed that multiple channel service quality includes three components: physical, virtual, and integrated service quality. Among them, MCIQ reflects the overall result of the multiple channel integration framework. MCIQ is defined as the ability to provide customers with a seamless service experience through multiple channels. It includes two components: service structure and interactive integration. The service structure is the quality of customer combined services (or service elements) and related channels. Interaction integration refers to the consistency of interaction between customers and channels among different channels, leading to a consistent service experience.

There are many classifications of comprehensive evaluation methods. The relationship between evaluation and use of information characteristics can be divided into the data-driven evaluation, model-driven evaluation, expert knowledge-driven evaluation, and evaluations based on data, models, and expert knowledge [8]. According to the theoretical basis on which each evaluation method is based, comprehensive evaluation methods are roughly divided into four categories.

2.1.1. Experts Grading Method. The main methods are the expert meeting method, Delphi method, and Gl method [9]. Such methods are simple to operate and can use experts' knowledge, and the conclusions are easy to use. The disadvantage is that the subjectivity is relatively strong, and the conclusion is difficult to converge in the evaluation of multiple people. It is suitable for decision-making analysis objects at the strategic level, and systems that cannot be quantified are difficult to quantify [10].

\subsubsection{Operations Research and Other Mathematical Methods}

(1) Data Envelopment Analysis Method. Evaluating the relative effectiveness of units of the same type is based on sex versus efficiency, based on multitarget input and multitarget output, and based on a set of criteria to determine the frontier of significant production [11]. It is possible to evaluate large systems with multiple inputs and multiple outputs and use "window" technology to find unit weaknesses and improve. The disadvantage is that it only shows the evaluation unit's relative development index and cannot show the actual development level. Applicable evaluation objects include evaluating the technology, scale effectiveness of the production function in economics, industry benefit evaluation, and the effectiveness of the education sector.

(2) Analytic Hierarchy Process Method. The working principle of the method is to target a multilevel structure system, determine multiple judgment matrices by comparing relative quantities, take the characteristic phasor corresponding to their characteristic roots as weights, and finally synthesize the total weights and sort them [12]. It is a method with relatively high reliability, a small error, and a wide range of applications. It has promising service quality evaluation applications, cost-benefit decision-making, resource allocation sequence, and conflict analysis [13].

(3) Fuzzy Comprehensive Evaluation Method. The fuzzy comprehensive evaluation method's working principle is to replace the number in the judgment matrix of the analytic hierarchy process with a fuzzy set by introducing a membership function and quantify the constraint conditions to perform mathematical solutions [14]. This method can overcome the shortcomings of the "unique solution" in the traditional method and obtain multiple problem solutions based on different possibilities. It has scalability and conforms to the "flexible management" idea in modern management [15]. It is suitable for consumer preference identification, decision-making expert systems, securities investment analysis, bank project loan object identification, quality evaluation, and safety evaluation [16]. 
(4) Gray Comprehensive Evaluation Method. This method selects the optimal value from the evaluated object's various indicators as the evaluation standard. It then compares and ranks the evaluated objects using the degree of similarity between each plan and the optimal plan [17]. In the gray comprehensive evaluation method, the data do not need to be normalized, and there is no need for many samples. The calculation is simple, and the reliability is substantial [18]. The disadvantage is that the sample data must have time series characteristics and have all the disadvantages of "relative evaluation." It is suitable for projects that deal with inadequate information systems and only have a small amount of observation data.

2.1.3. Intelligent Evaluation Method. The intelligent evaluation method is represented by the artificial neural network evaluation method, an artificial neural network technology that simulates the human brain's intelligent processing process. It can "learn or be trained to acquire knowledge" through the BP algorithm. It stores the acquired knowledge in the neuron's weight and reproduces the relevant information through association [19]. It can "compute" and "refine" the objective laws of the evaluation object itself and to evaluate the evaluation objects of the same attribute [20]. The method's advantage is that the network has selfadaptability and fault tolerance and can handle large and complex systems with nonlinearity, nonlocality, and nonconvexity. The disadvantage is that the accuracy is not high, and many training samples are required. Its application areas continue to expand, involving bank loan projects, stock price evaluation, and evaluation of the comprehensive urban development [21].

\subsubsection{Statistical Methods}

(1) Principal Component Analysis Method. This method's working principle is based on the common elements that dominate the existence of related economic variables. The goal is to study the correlation matrix of the original variables' internal structure and find several irrelevant comprehensive evaluation indicators that affect a specific economic process to represent the original variables. The principal component analysis method is comprehensive, comparable, objective, and reasonable. The disadvantage is that the actual meaning of new variables is often challenging to figure out, and the calculation process is cumbersome. It is suitable for evaluation items that classify evaluation objects, which have many evaluation indicators and have complex relationships between indicators.

(2) Cluster Analysis Method. Cluster analysis is an evaluation method for systematic clustering that calculates the distance between indicators or the similarity coefficient. It can solve the evaluation objects with a large degree of correlation. The disadvantage is that it requires a large amount of statistical data and does not reflect the objective development level. It

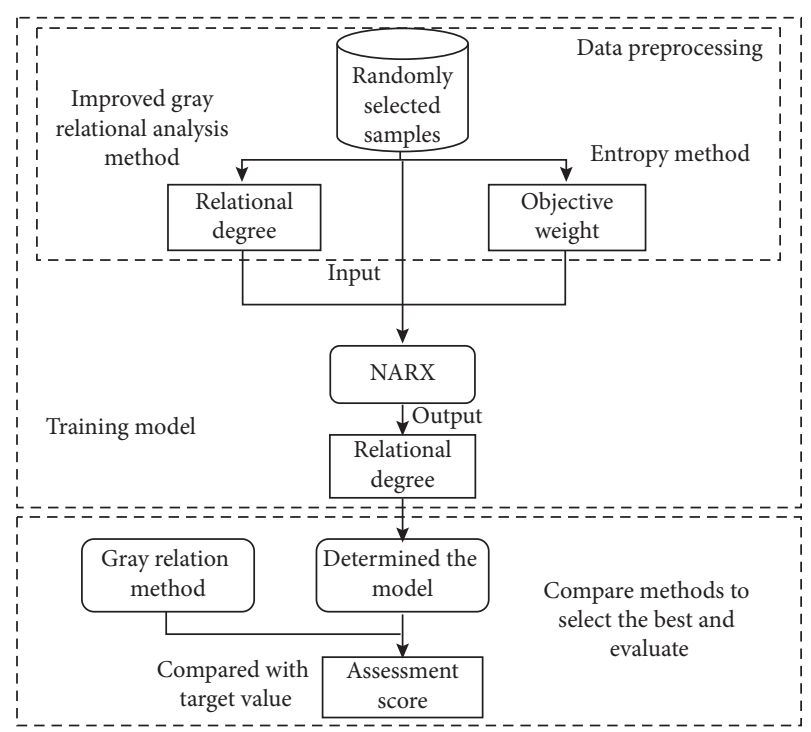

Figure 1: Methodology.

is suitable for portfolio investment selection and regional development level evaluation.

Although there are many mature methods and models for integrated quality evaluation, as mentioned above, they all have their limitations. This paper uses a NARX neural network to overcome low accuracy in general intelligent evaluation methods and the need for a large number of training samples. The improved GRA method and entropy method are used to preprocess the sample data so that the MCIQ can have higher accuracy.

\section{Methodology}

As shown in Figure 1, this research is mainly divided into four parts:

(1) Determine the objective weight value of each indicator based on the entropy method.

(2) The improved GRA method is used to obtain a comprehensive gray relational degree.

(3) The NARX model is trained with the dataset processed by the GRA method. Then, the trained NARX model is used to predict the comprehensive gray relational degree.

(4) The result calculated by the GRA method and NARX model is standardized and compared with the target value. Finally, the quantitative results of MCIQ are given.

Each indicator's objective weight can be obtained through the entropy method, which is based on the score and standardized as the target value. The improved GRA method is used to associate the standard sample of each evaluation indicator constructed with the sample to be tested. Then, the comprehensive gray relational degree of the index is obtained, and the improved comprehensive gray relational degree is used as the output of the NARX model. 
Next, the evaluation index data in the sample to be tested are used as the input to the NARX model to train the NARX neural network. Then, the trained NARX model is used to predict and obtain the gray relational degree. Because each index's weight will be obtained in calculating the comprehensive gray relational degree, it can be standardized to obtain the sample quality evaluation score. Then, the results obtained by NARX and GRA are compared with the target value. The result closer to the target value is better and more accurate. Finally, the results of the MCIQ evaluation are obtained. These results provide new ideas and theoretical guidance for traditional retailers to use the advantages of multiple channels to expand their businesses.

\section{Multiple Channel Integration Quality Evaluation Model Using NARX}

4.1. Comprehensive Evaluation Index System Construction. This research divides the retailer's MCIQ into 8 dimensions from the perspective of customer perception, as shown in Table 1.

A comprehensive evaluation index for MCIQ contains the following dimensions: process consistency $x_{1}$, information consistency $x_{2}$, emotional value $x_{3}$, procedural value $x_{4}$, service structure transparency $x_{5}$, online result value $x_{6}$, business relevance $x_{7}$, and online purchase intention $x_{8}$.

\subsection{MCIQ Data Processing}

4.2.1. Improved GRA. The GRA is carried out by identifying the degree of similarity or difference between system factors' development trends. The GRA method can analyze the correlation between the standard samples of the above eight indicators and the samples to be evaluated to obtain the comprehensive gray relational degree of the indicators [22]. The main steps are as follows:

(1) Constructie the original evaluation matrix for gray correlation evaluation:

$$
X=\left[\begin{array}{c}
X_{1} \\
X_{2} \\
\vdots \\
X_{i} \\
\vdots \\
X_{m}
\end{array}\right]=\left[\begin{array}{cccccc}
X_{11} & X_{12} & \cdots & X_{1 j} & \cdots & X_{1 n} \\
X_{21} & X_{22} & \cdots & X_{2 j} & \cdots & X_{2 n} \\
\vdots & \vdots & \vdots & \vdots & \vdots & \vdots \\
X_{i 1} & X_{i 2} & \cdots & X_{i j} & \cdots & X_{j n} \\
\vdots & \vdots & \vdots & \vdots & \vdots & \vdots \\
X_{m 1} & X_{m 2} & \cdots & X_{m j} & \cdots & X_{m n}
\end{array}\right] \text {, }
$$

where $x_{i j}$ represents the $j$-th index of the $i$-th object and the original evaluation matrix is composed of $m$ objects and $n$ indexes. The reference number is $X_{0}=\left[\begin{array}{llllll}X_{00} & X_{01} & \ldots & X_{0 j} & \ldots & X_{0 n}\end{array}\right]$.

(2) Standardize data processing and establish an evaluation absolute difference matrix:
Calculate the absolute value of the difference between each element of the original evaluation matrix and its corresponding element in the reference sequence denoted as the following equation:

$$
\left[\Delta_{k}\right]=\left(X_{k}-X_{0}\right), \quad k=1,2, \ldots, n .
$$

(3) Calculate each index's gray relational degree between the sample to be evaluated and the standard sample [23] and transform the absolute difference matrix in equation (2). The correlation coefficient is shown in equation (2):

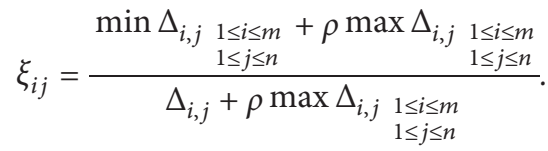

In equation (3), $\rho$ is the resolution coefficient.

This paper adopts the method of dynamic value selection to select the resolution coefficient. Let $\Delta_{v}(k)=$ $\left(\left[\Delta_{k}\right] / m\right)$, then $\varepsilon(k)=\left(\Delta_{v}(k) / \max _{i} \max _{k}\left[\Delta_{k}\right]\right)$, $\rho=\{\rho(1), \rho(2), \ldots, p(n)\}$. The value of $\rho(k)$ is as follows: when $(1 / \varepsilon(k))>3$, take $\rho(k)=1.5 \varepsilon(k)$; when $2 \leq(1 / \varepsilon(k)) \leq 3$, take $\rho(k)=2 \varepsilon(k)$; when $0<$ $(1 / \varepsilon(k))<2, \rho(k)=0.9$; when $\varepsilon(k)=0$, and $\rho(k)=$ arbitrary value.

(4) Compare the sample indicators to be evaluated and the indicators in the standard sample one by one. Then, calculate the correlation coefficient $\xi_{i j}$ and the indicator weight $\omega_{j}$ according to formula (3). Then, the comprehensive gray relational degree of the sample is evaluated and compared with the ideal sample gray relational degree as shown in the following equation:

$$
\Phi_{i}=\sum_{j=1}^{n} \omega_{j} \xi_{i}(j), \quad \Phi_{i} \in[0,1]
$$

where $i=2,3, \ldots, m ; j=1,2, \ldots, n$; and $\omega_{j}$ is the weight of the $j$-th index.

4.2.2. The Entropy Method. The process of using the entropy method to determine the objective weight of the index is as follows: in an evaluation problem with $n$ evaluation indexes and $m$ evaluation levels, the entropy value of the $i$-th individual evaluation index is defined as the following equation [24]:

$$
H_{i}=-\frac{1}{\ln m} \sum_{j=1}^{m} f_{i j} \ln f_{i j}, \quad i=1,2, \ldots, n,
$$

where $f_{i j} \neq 0$, when $f_{i j}=0, f_{i j} \ln f_{i j}=0$.

The entropy weight of the $i$-th evaluation index is defined in the following equation: 
TABLE 1: Retailer's MCIQ into 8 dimensions.

\begin{tabular}{|c|c|}
\hline Index & Meaning \\
\hline Process consistency & $\begin{array}{l}\text { It refers to the consistency of process attributes compared between different channels, such as service } \\
\text { perception, image, waiting time, and customer service level. }\end{array}$ \\
\hline $\begin{array}{l}\text { Information consistency } \\
\text { Emotional value }\end{array}$ & $\begin{array}{l}\text { It refers to the consistency of response information obtained by consumers through different channels. } \\
\text { It refers to the measurement of shopping emotion. For example, online store shopping is exciting. }\end{array}$ \\
\hline Procedural value & $\begin{array}{l}\text { It refers to measuring the purchase process ease. For example, it is more convenient to shop in the online } \\
\text { retailer store. }\end{array}$ \\
\hline $\begin{array}{l}\text { Transparency of service } \\
\text { structure }\end{array}$ & $\begin{array}{l}\text { It refers to the customer's perception of the attributes of all available channels. It will directly affect the } \\
\text { choice of channels for customers to receive specific services. The transparency of the service structure } \\
\text { reflects the level of the retailer's multiple channel service structure, the consistency of information, and } \\
\text { business relevance. Process consistency represents the three forms of interaction and integration between } \\
\text { channels. }\end{array}$ \\
\hline Online result value & $\begin{array}{l}\text { It refers to the measurement of the final result of online purchases, such as the value of the retailer's online } \\
\text { store's goods and services. }\end{array}$ \\
\hline Business relevance & $\begin{array}{c}\text { It refers to the connection between interaction generated through a specific channel and interaction } \\
\text { generated through other channels. }\end{array}$ \\
\hline Online purchase intention & It is the willingness of consumers to buy online. \\
\hline
\end{tabular}

$$
\omega_{i}=\frac{1-H_{i}}{n-\sum_{i=1}^{n} H_{i}},
$$

meeting the conditions $\sum_{i=1}^{n} \omega_{i}=1,0 \leq \omega_{i} \leq 1$.

The calculated $W=\left\{\omega_{1}, \omega_{2}, \ldots, \omega_{n}\right\}$ is the objective weight value of each index determined by the entropy method.

4.3. Building the NARX Model. The NARX neural network, as a dynamic recurrent neural network with output feedback connection, can effectively overcome the phenomenon of error accumulation in time series data. Therefore, this article uses a nonlinear autoregressive dynamic neural network model for processing. This model belongs to a commonly used method in time series analysis [25]. It uses the combination of variables at several previous moments to infer the development of variables at subsequent moments. The process of NARX neural network model building is shown in Figure 2.

A typical NARX neural network comprises an input layer, hidden layer, output layer, and input delay function. Its basic structure is shown in Figure 3. In the figure, $X(k)$ represents the neural network's input value and $Y(k)$ represents the output value of the neural network.

The difference between a NARX neural network and the ordinary neural network adds a delay function before the hidden layer. The effect of the delay function on the output is as follows [26]:

$$
y(t)=f(x(t-1), \ldots, x(t-d), y(t-1), \ldots, y(t-d)),
$$

where $d$ represents the delay order.

In the NARX neural network, the output signal is delayed and then input into the neural network, and the hidden layer and the output layer are combined to obtain the final output result [27]. $i$ represents the number of input data, $l$ is the number of hidden layer neurons, $x_{i}$ represents the $i$-th input signal of the network, $w_{i j}$ represents the connection weight between the $i$-th output delay signal and

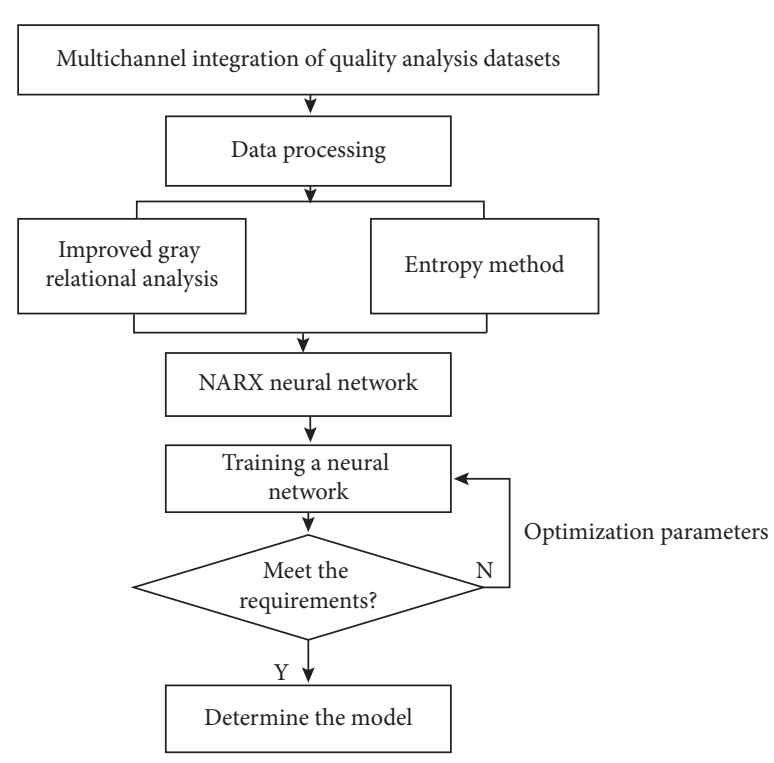

FIGURE 2: Flowchart of NARX neural network model setting.

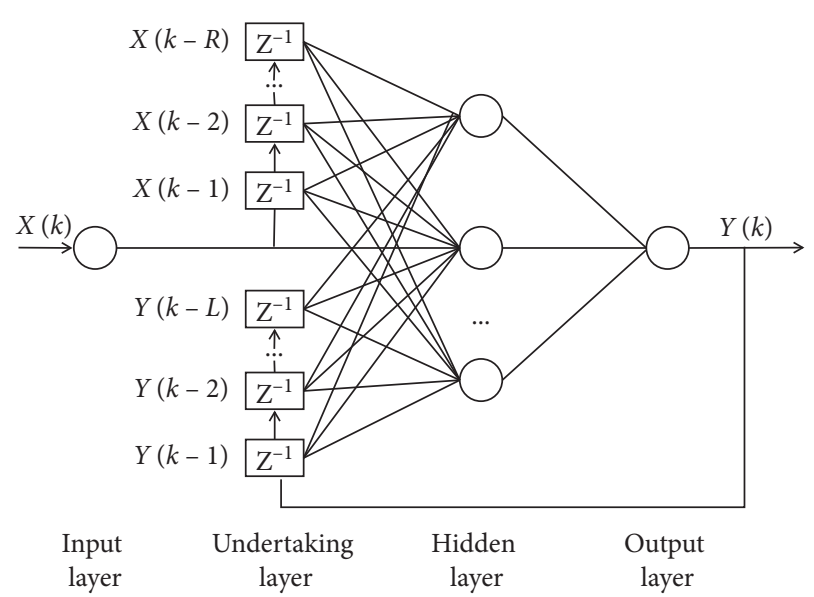

Figure 3: Schematic diagram of NARX neural network structure. 
the $j$-th neuron, $a_{j}$ represents the threshold value of the $j$-th hidden layer neuron, and the hidden layer activation function $f$ is integrated to obtain the calculation result of each neuron as shown in the following equation [28]:

$$
H_{j}=f\left(\sum_{i=1}^{n} w_{i j} x_{i}+a_{j}\right) \text {. }
$$

Using $w_{i j}$ as the connection weight between the $j$-th neuron in the hidden layer and the output layer neuron [29] and $b$ as the output layer neuron threshold, calculate the output as follows:

$$
O=f\left(\sum_{j=1}^{l} H_{j} w_{j}+b\right) .
$$

\section{Multiple Channel Integration Quality Assessment}

5.1. Evaluation of Data Collection. According to the comprehensive evaluation index system of multisource channel integration quality determined above, fifty thousand consecutive samples of a commodity in 3 months are used as a dataset. The data fields in the dataset are the consistency of the collection process $x_{1}$, information consistency $x_{2}$, emotional value $x_{3}$, procedural value $x_{4}$, service structure transparency $x_{5}$, online result value $x_{6}$, business relevance $x_{7}$, and online purchase intention $x_{8}$. Table 2 shows sample records in the evaluation datasheet.

5.2. Evaluation Using Improved GRA Method. The GRA method is widely used, but there are still some defects. The resolution coefficient $\rho$ is usually set to 0.5 based on experience or uniformly set to 0.5 . This paper adopts the method of determining the resolution coefficient's dynamic state to improve the GRA method. According to the data series's stability, this paper adopts the method of dynamic value selection to select the resolution coefficient. Fifty thousand consecutive samples of a product for three months are selected as the dataset to verify the method. Compared with the traditional gray correlation method, the improved gray relational degree suppresses outliers' influence in the observation sequence on the correlation space, making the correlation analysis more realistic. Figure 4 shows the weight of the evaluation index.

5.3. Comprehensive Evaluation Using NARX Neural Network. This NARX model takes process consistency $x_{1}$, information consistency $x_{2}$, emotional value $x_{3}$, procedural value $x_{4}$, service structure transparency $x_{5}$, online result value $x_{6}$, business relevance $x_{7}$, and online purchase intention $x_{8}$ as input. The comprehensive improvement in the gray relational degree $x_{9}$ is the output of the NARX
TABle 2: Evaluation data.

\begin{tabular}{lcccccccc}
\hline Sample number & $x_{1}$ & $x_{2}$ & $x_{3}$ & $x_{4}$ & $x_{5}$ & $x_{6}$ & $x_{7}$ & $x_{8}$ \\
\hline 1 & 3.0 & 1.0 & 1.0 & 3.0 & 1.0 & 5.0 & 489.0 & 0.0417 \\
2 & 1.0 & -1.0 & -1.0 & 4.0 & 1.0 & 4.0 & 30.0 & 0.0376 \\
3 & 1.0 & 2.0 & 1.0 & 3.0 & 0.0 & 1.0 & 545.0 & 0.0000 \\
$\vdots$ & $\vdots$ & $\vdots$ & $\vdots$ & $\vdots$ & $\vdots$ & $\vdots$ & $\vdots$ & $\vdots$ \\
50000 & -1.0 & 1.0 & 1.0 & 2.0 & 0 & 4.0 & 244.0 & 0.0000 \\
\hline
\end{tabular}

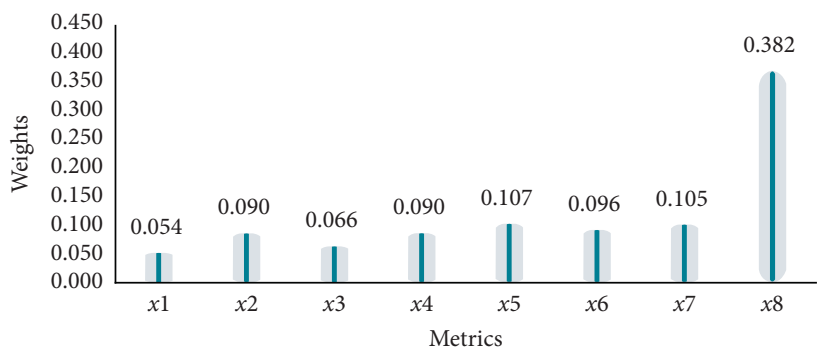

FIgURE 4: Evaluation index weight diagram.

TABLE 3: Training results.

\begin{tabular}{lccc}
\hline Result & Target & $\mathrm{MSE} / 10^{-7}$ & $R / 10^{-1}$ \\
\hline Train & 35000 & 1.53876 & 9.96358 \\
Verify & 15000 & 1.69412 & 9.95800 \\
Test & 15000 & 1.56598 & 9.96437 \\
\hline
\end{tabular}

model. The input data are inputted into the NARX neural network for training. The network training method uses the Levenberg-Marquardt algorithm, the number of hidden layer neurons is set to 10 , and the delay is set to 2 . It can be seen from Table 3 and Figure 5 that the network has an excellent fitting effect, so the training of this model is ideal.

5.4. Comparison of Evaluation Methods. The MSE of the GRA method is 0.0000153, and the MSE of the NARX neural network method is 0.000000180 . It can be seen from Figure 6 that the output value produced by the NARX neural network is closer to the target output value, while the output error of the GRA method is more massive. Therefore, NARX neural network comprehensive evaluation is more applicable, more accurate, and can more effectively reflect isolated networks' autonomous operation capability.

5.5. Multiple Channel Integration Quality Capability Score. The evaluation results with standardized scores between [1, $0.75)$ are regarded as excellent, $[0.75,0.5)$ as medium, $[0.5$, $0.25)$ as qualified, and $[0.25,0)$ as unqualified. Selecting a group of typical samples as the reference samples for MCIQ capability evaluation, the evaluation results are shown in Table 4. 


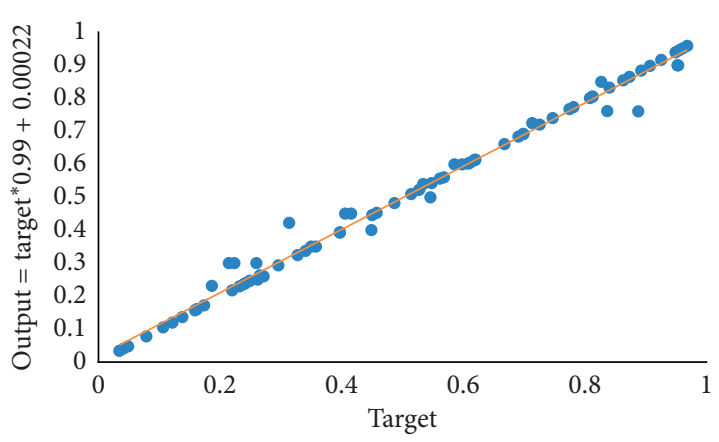

(a)

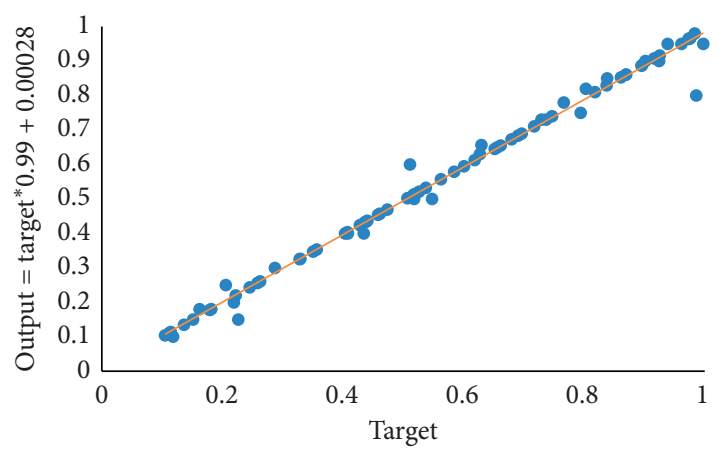

(c)

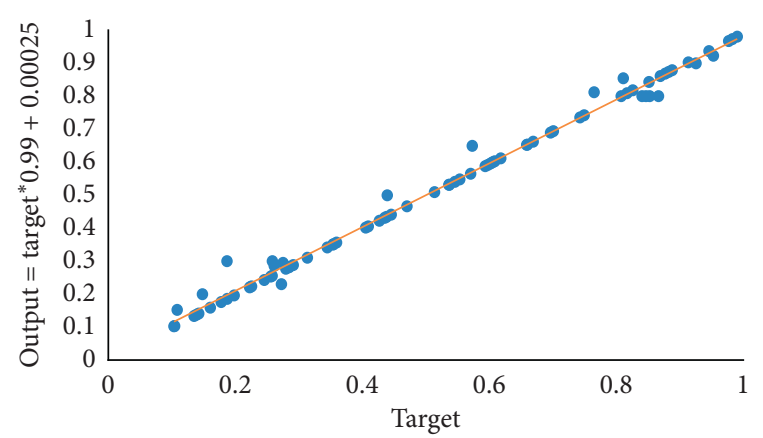

(b)

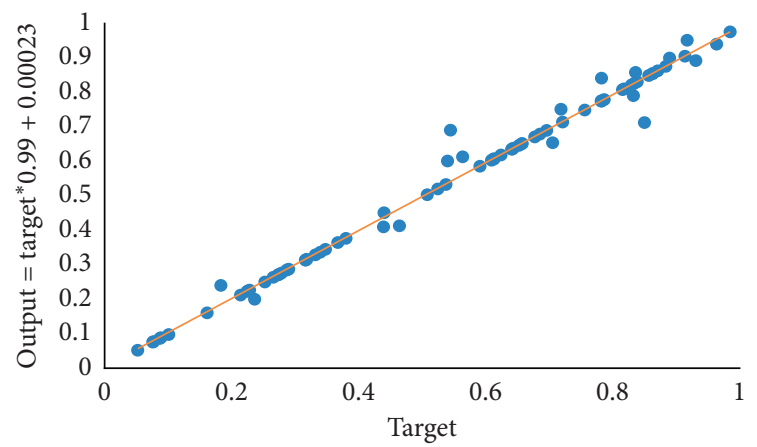

(d)

Figure 5: Neural network model fitting diagram: (a) $R=0.9959$, (b) $R=0.99435$, (c) $R=0.99464$, and (d) $R=0.99518$.

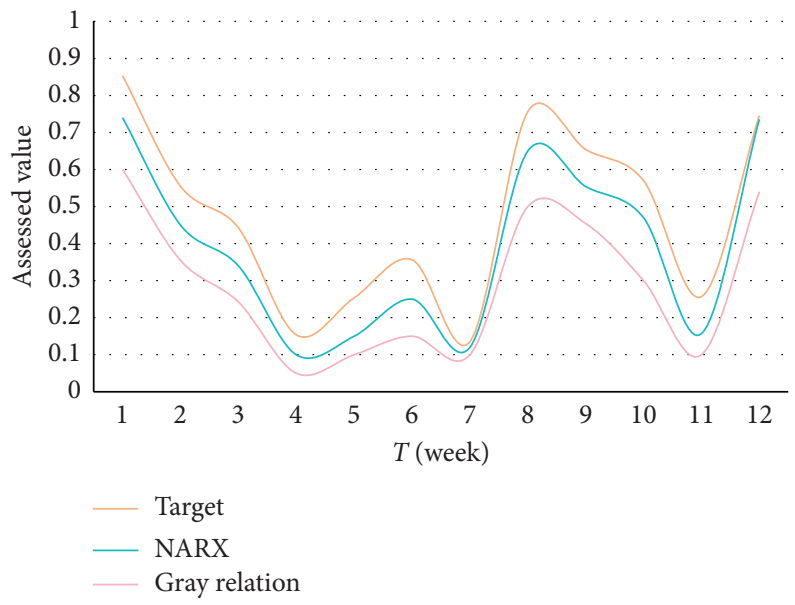

Figure 6: Comparison of evaluation results and target values.

TABLE 4: Evaluation results of typical samples.

\begin{tabular}{lccc}
\hline Reference sample sequence & $t$ (week) & Normalized score & Evaluation result \\
\hline 1 & 1 & 0.8532 & Excellent \\
2 & 3 & 0.5547 & Medium \\
3 & 6 & 0.4426 & Qualified \\
4 & 4 & 0.1536 & Unqualified \\
\hline
\end{tabular}

\section{Conclusions and Future Work}

This paper proposes a method for a comprehensive evaluation of MCIQ capability using the NARX neural network.
This method's MCIQ index system comprehensively considers the impact of key factors affecting online purchase intentions based on analyzing multiple channel integration. The entropy method and the advantages of the improved 
GRA method are used in the data processing. Then, NARX neural network modeling is used to avoid the disadvantages of the GRA algorithm. The evaluation results of multiple scenarios verify that this method is effective and applicable.

Although this study has made a certain breakthrough in the quality assessment of multiple channel integration, the results still have some limitations. This research has not considered shoppers' characteristics, such as shopping orientation, involvement, and time pressure. Future research can further explore the influence of these factors on the model.

\section{Data Availability}

The data used to support the findings of this study are available from the corresponding author upon request.

\section{Conflicts of Interest}

The authors declare that they have no conflicts of interest.

\section{References}

[1] B. Berman and S. Thelen, "A guide to developing and managing a well-integrated multi-channel retail strategy," International Journal of Retail \& Distribution Management, vol. 32, no. 3, pp. 147-156, 2004.

[2] K. A. Saeed, V. Grover, and Y. Hwang, "Creating synergy with a clicks and mortar approach," Communications of the ACM, vol. 46, no. 12, pp. 206-212, 2003.

[3] C. M. Chan and S. L. Pan, Intertwining Offline and Online Channels in Multiple Channel Public Service Delivery: A Case Study, Academy of Management, Briarcliff Manor, NY, USA, 2005.

[4] H.-H. Lee and J. Kim, "Investigating dimensionality of multichannel retailer's cross-channel integration practices and effectiveness: shopping orientation and loyalty intention," Journal of Marketing Channels, vol. 17, no. 4, pp. 281-312, 2010.

[5] L.-B. Oh and H.-H. Teo, "Consumer value co-creation in a hybrid commerce service-delivery system," International Journal of Electronic Commerce, vol. 14, no. 3, pp. 35-62, 2010.

[6] R. Sousa and C. A. Voss, "Service quality in multichannel services employing virtual channels," Journal of Service Research, vol. 8, no. 4, pp. 356-371, 2006.

[7] R. Madaleno, H. Wilson, and R. Palmer, "Determinants of customer satisfaction in a multi-channel B2B environment," Total Quality Management \& Business Excellence, vol. 18, no. 8, pp. 915-925, 2007.

[8] T. M. T. Hossain, S. Akter, U. Kattiyapornpong, and Y. K. Dwivedi, "Multichannel integration quality: a systematic review and agenda for future research," Journal of Retailing and Consumer Services, vol. 49, pp. 154-163, 2019.

[9] L. M. Wang, Z. Y. Hao, X. M. Han, and R. H. Zhou, "Gravity theory-based affinity propagation clustering algorithm and its applications," Tehnicki Vjesnik-Technical Gazette, vol. 25, no. 4, pp. 1125-1135, 2018.

[10] J. Niresh, N. Archana, and G. Anand Raj, "Optimisation of linear passive suspension system using MOPSO and design of predictive tool with artificial neural network," Studies in Informatics and Control, vol. 28, no. 1, pp. 105-110, 2019.
[11] J. Li, S. X. Pan, L. Huang, and X. Zhu, "A machine learning based method for customer behavior prediction," Tehnicki Vjesnik-Technical Gazette, vol. 26, no. 6, pp. 1670-1676, 2019.

[12] D.-V. Căiman and T.-L. Dragomir, "Empirical voltage-current signatures for individual household consumers obtained by non-linear regression," Studies in Informatics and Control, vol. 28, no. 2, pp. 201-212, 2019.

[13] Y. Ge and H. Wu, "Prediction of corn price fluctuation based on multiple linear regression analysis model under big data," Studies in Informatics and Control, vol. 28, 2019.

[14] C. Ciurea and F. G. Filip, "Virtual exhibitions in cultural institutions: useful applications of informatics in a knowledge-based society," Studies in Informatics and Control, vol. 28, no. 1, pp. 55-64, 2019.

[15] K.-S. Moon and H. Kim, "Performance of deep learning in prediction of stock market volatility," Economic Computation and Economic Cybernetics Studies And Research, vol. 53, pp. 77-92, 2019.

[16] X.-L. Shen, Y.-J. Li, Y. Sun, and N. Wang, "Channel integration quality, perceived fluency and omnichannel service usage: the moderating roles of internal and external usage experience," Decision Support Systems, vol. 109, pp. 61-73, 2018.

[17] C. Zehir and E. Narcıkara, "E-service quality and e-recovery service quality: effects on value perceptions and loyalty intentions," Procedia-Social and Behavioral Sciences, vol. 229, no. 427, p. 43, 2016.

[18] J. Atanasijevic and D. Milosevic, "Upgrading the business intelligence system by implementing the decision tree model in the R software package," Studies in Informatics and Control, vol. 29, no. 2, pp. 243-254, 2020.

[19] I. Vrecko, J. Kovac, B. Rupnik, and B. Gajsek, "Using queuing simulation model in production process innovations," International Journal of Simulation Modelling, vol. 18, no. 1, pp. 47-58, 2019.

[20] M. S. Yang, L. Ba, Y. Liu et al., “An improved genetic simulated annealing algorithm for stochastic two-sided assembly line balancing problem," International Journal of Simulation Modelling, vol. 18, no. 1, pp. 175-186, 2019.

[21] L. Lei, W. Chen, Y. Xue, and W. Liu, "A comprehensive evaluation method for indoor air quality of buildings based on rough sets and a wavelet neural network," Building and Environment, vol. 162, 2019.

[22] C.-T. Su and F.-F. Wang, "Integrated fuzzy-connective-based aggregation network with real-valued genetic algorithm for quality of life evaluation," Neural Computing and Applications, vol. 21, no. 8, pp. 2127-2135, 2012.

[23] J. Zhao, G. Ji, Y. Tian, Y. Chen, and Z. Wang, "Environmental vulnerability assessment for mainland China based on entropy method," Ecological Indicators, vol. 91, pp. 410-422, 2018.

[24] H.-Y. Wu, a. Tsai, and H.-S. Wu, "A hybrid multi-criteria decision analysis approach for environmental performance evaluation: an example of the TFT-LCD manufacturers in Taiwan," Environmental Engineering and Management Journal, vol. 18, no. 3, pp. 597-616, 2019.

[25] J. Li, U. Konuş, F. Langerak, and M. C. D. P. Weggeman, "Customer channel migration and firm choice: the effects of cross-channel competition," International Journal of Electronic Commerce, vol. 21, no. 1, pp. 8-42, 2017.

[26] B. Xue, M. Liu, and Q. Sun, "Applications of grey relational analysis to enterprise performance evaluation of express listed companies in China," in Proceedings of the 2017 3rd 
International Forum on Energy, Environment Science and Materials (IFEESM 2017), Shenzhen, China, November 2017.

[27] A. Coser, M. M. Maer-Matei, and C. Albu, "Predictive models for loan default risk assessment," Economic Computation And Economic Cybernetics Studies And Research, vol. 53, pp. 149-165, 2019.

[28] E. Comăniţă, P. Cozma, I. Simion, M. Roșca, and M. Gavrilescu, "Evaluation of eco-efficiency by multicriteria decision analysis. case study of eco-innovated and ecodesigned products from recyclable waste," Environmental Engineering and Management Journal, vol. 17, pp. 1791-1804, 2018.

[29] P. P. Dey, S. Pramanik, and B. C. Giri, "An extended grey relational analysis based multiple attribute decision making in interval neutrosophic uncertain linguistic setting," $\mathrm{Neu}$ trosophic Sets and Systems, vol. 11, pp. 21-30, 2016. 